\title{
Synthesis and in vitro antitumor activity of novel naphthyridinone derivatives
}

\author{
Xue-Dong Jia ${ }^{\text {a, b }}$, Shuo Wang ${ }^{\text {a }}$, Ming-Hua Wang ${ }^{\text {a }}$, Ming-Liang Liu ${ }^{\text {a, }}$, Gui-Min Xia ${ }^{\text {a }}$, Xiu-Jun Liu ${ }^{\text {a }}$, Yun \\ Chai ${ }^{\text {a }}$, Hong-Wei He ${ }^{\text {a, * }}$ \\ ${ }^{a}$ Institute of Medicinal Biotechnology, Chinese Academy of Medical Sciences and Peking Union Medical College, Beijing 100050, China \\ ${ }^{b}$ The First Affiliated Hospital of Zhengzhou University, Zhengzhou 450052, China
}

\section{ARTICLE INFO}

\section{ABSTRACT}

\section{Article history:}

Received 6 June 2016

Received in revised form 4 July 2016

Accepted 12 July 2016

Available online

\section{Keywords:}

Naphthyridinones

Synthesis

Antitumor activity

Voreloxin

\begin{abstract}
A series of naphthyridinone derivatives based on 1a (a precursor of Voreloxin) were designed and synthesized. Seven compounds having $>70 \%$ inhibition against HL60 at $30 \mu \mathrm{mol} / \mathrm{L}$ were further evaluated for their in vitro antitumor activity by SRB assay. Results reveal that thiazol-2yl and 3-aminomethyl-4-benzyloxyimino-3-methylpyrrolidin-1-yl groups are optimal at the N-1 and C-7 positions of naphthyridinone core, respectively. 10j exhibits broad-spectrum activity $\left(\mathrm{IC}_{50}:<0.5-6.25 \mu \mathrm{mol} / \mathrm{L}\right)$ against all of the tested cell lines including Etoposide- and/or 1aresistant ones, and is 1.3 -fold to $>100$-fold more potent than the two references against eight of these cell lines.
\end{abstract}

\section{Introduction}

Cancers still remain the first and second leading causes of death in economically developed and developing countries, respectively [1,2]. It is expected that annual cancer cases will rise from 14 million in 2012 to 22 within the next 2 decades [3]. Encouragingly, substantial progress has been made to develop more effective and safer strategies for treating cancers, and many classes of antitumor agents with diverse novel structures have been introduced in the market or under development in the past decade [4]. However, there continues to be a need for new antitumor drugs from either novel scaffolds or further structural modifications of existing drugs.

Quinolones represent an extremely successful family of antibiotics that have a broad-spectrum antibacterial activity and relatively few side effects [5]. Among of them, ciprofloxacin, ofloxacin and levofloxacin are also used as second-line drugs to treat tuberculosis [6]. Recently, quinolones as "privileged building blocks" have been reported to display many "nonclassical" biological profiles, such as antitumor, anti-HIV-1 integrase, anti-HCV-NS3 helicase and -NS5B-polymerase activities [7,8]. It's exciting that Voreloxin (Fig. 1) with a broad-spectrum antitumor activity [9] the first quinolone antitumor drug, was approved as an orphan drug for the treatment of acute myeloid leukemia by the US FDA in 2009, and phase I-III clinical trials in patients with various tumors are currently ongoing [10].

Fig. 1. Chemical structures of Voreloxin and 1a

In the course of our search for more potent antitumor agents, we focused our interest on structural modifications of Voreloxin. Given that 3-aminopyrrolidine, but not (3S, 4S)-3-methoxy-4-(methylamino)pyrrolidine, is commercially available, compound 1a (Fig. 1) possessing the in vitro antitumor activity comparable to Voreloxin [11] was chosen as the lead compound in our work. First, the thiazoly2-yl group at the N-1 position of $1 \mathbf{a}$ was replaced with its isosteres (1H-imidazol-2-yl, oxazol-2-yl, etc.) to examine whether it is the optimal group of this position. On the other hand, our previous works have emphasized the importance of an oxime functional moiety of the C-7 side chain with respect to biological activities of quinolones [12-15]. Thus, a four, five- or six-membered nitrogen

* Corresponding authors.

E-mail addresses: 1mllyx@126.com (M.-L. Liu), hehwei@imb.pumc.edu.cn (H.-W. He) 
heterocycle containing an alkoxyimino group was introduced at the C-7 position instead of the 3-aminopyrrolidin-1-yl one of 1a, to identify if a nitrogen heterocycle with an oxime moiety is permitted at this position. Our primary objective was to optimize the potency of these compounds against human cancer cell lines. A simple structure-activity relationship (SAR) study was also explored to facilitate the further development of the naphthyridinone derivatives.

\section{Experimental}

\subsection{Synthetic chemistry}

To study the effect of various substituents at the N-1 position of the 7-(3-aminopyrrolidin-1-yl)naphthyridinone core, eight analogues of 1a were designed. A synthetic route to these compounds is illustrated in Scheme 1. The key intermediates 6a-i were readily prepared from 2, 6-dichloronicotinic acid (2) via a five-step procedure [14,16]. Hydrolysis of the core esters 6a-d in $\mathrm{HCl}-\mathrm{HOAc}$ followed by condensation with 3- $N$-Boc-aminopyrrolidine in the presence of triethylamine gave 8a-d. However, the esters $6 \mathbf{e}-\mathbf{i}$ were not converted to the corresponding acids in a similar manner as for preparation of 8a-d. After various attempts, 8e-i were successfully obtained from 6e-i by condensation with the side chain compounds and then hydrolysis of the resulted esters 7e-i. The Boc-protecting group of 8a-i was removed by hydrogen chloride gas in dichloromethane to yield the desired naphthyridinone derivatives $\mathbf{1 a - i}$ as hydrochloric acid salts.

Scheme 1. Synthesis of 7-(3-aminopyrrolidin-1-yl)naphthyridinone derivatives 1a-i.

The synthetic route of novel 1-(thiazol-2-yl)naphthyridinones 10a-n containing nitrogen heterocycles with an alkoxyimino group was depicted in Scheme 2. Condensation of $6 \mathbf{a}$ with various side chain compounds[12,17-19] and then hydrolysis of the resulted esters 9a-n yielded 10a-n. 
Since the oxime group can exist in the $E$ or $Z$ configuration, it was necessary to determine the geometries of all the oxime target compounds 10a-n. Preparing X-ray quality single crystals of any oxime intermediate or product met with no success in this study, but the oxime geometry would be expected to have the E-configuration according to the data in published papers [20,21]. The concrete synthetic procedures, the physical characteristics, and ${ }^{1} \mathrm{H}$ NMR for all the synthesized compounds are listed in the Supporting information.

\subsection{Anti-tumor activities}

All the synthesized target compounds were preliminarily investigated for their in vitro activity against HL60 (leukemia) at the concentration of $30 \mu \mathrm{M}$ by SRB (Sulforhodamine B) assay [22]. And the compounds having $>70 \%$ inhibition were subjected to IC $_{50}$ (50\% inhibition concentrations) determination against ten human cancer cell lines, including HL60, HepG2 (liver carcinoma), HCT116 (colon cancer), A549 (lung adenocarcinoma), PANC-1 (pancreatic carcinoma), Hela (cervical cancer), DU145 (prostatic cancer), SKOV3 (ovarian carcinoma), MCF-7 (breast cancer) and MCF-7/DOX (Doxorubicin-resistant MCF-7) by SRB assay.

\section{Results and discussion}

The inhibitory effects of the 7-(3-Aminopyrrolidin-1-yl) naphthyridinone derivatives 1a-i and 1-(thiazol-2-yl)naphthyridinone derivatives 10a-n were evaluated and listed in Table 1 and Table 2. Unfortunately, contrary to our predictions, the results indicated that all the newly synthesized 7-(3-Aminopyrrolidin-1-yl) naphthyridinone derivatives 1b-i (inhibition rates: 0-28\%) were much less active than the reference 1a $(75 \%$, Table 1$)$. Therefore, our modifications are not effective, and the thiazol-2-yl group is optimal for the N-1 position.

The $\mathrm{IC}_{50}$ values were compared with those of Etoposide and 1a (Table 2). The selected 1-(thiazol-2-yl)naphthyridinones have potent activity against these tested human cancer cell lines. All of them $\left(\mathrm{IC}_{50}:<0.5-49.23 \mu \mathrm{mol} / \mathrm{L}\right)$ are more active or comparable to Etoposide and 1a $\left(\mathrm{IC}_{50}\right.$ : 1.17->50 $\left.\mu \mathrm{mol} / \mathrm{L}\right)$ against HCT-116, A549, SKOV3 and MCF-7/DOX. Contrary to 1a, compounds 10h-j show significantly better activity against MCF-7/DOX $\left(\mathrm{IC}_{50}:<0.5 \mu \mathrm{mol} / \mathrm{L}\right)$ than MCF-7 ( $\left.\mathrm{IC}_{50}: 5.57-31.04 \mu \mathrm{mol} / \mathrm{L}\right)$. Moreover, 10j was found to have a broad-spectrum activity $\left(\mathrm{IC}_{50}:<0.5-6.25 \mu \mathrm{mol} / \mathrm{L}\right.$ ) against all of the tested cell lines including Etoposide- and/or 1a - resistant ones, and is 1.3 -> 100 fold more potent than those of the two references against these cell lines, except HL60 and HepG2.

According to the biological evaluation results shown in Tables 1 and 2, the antitumor activity of the naphthyridinone derivatives in this study depends on both of the groups at the N-1 and C-7 positions. The relative contribution of the heteroaromatic ring at the N-1 position to activity is as follows: thiazol-2-yl (1a) $>1 H$-imidazol-2-yl (1f) $>$ thiophen-3-yl (1d), and the others $(\mathbf{1 b}, \mathbf{1 c}, \mathbf{1 e}, \mathbf{1 g}-\mathbf{i})$ have no activity (Table 1 ).

On the other hand, 1-(thiazol-2-yl)naphthyridinones 10a-n generally exhibit in vitro activity (Table 1), which indicates that a nitrogen heterocycle with an oxime group is permitted at the C-7 position. Although a simple SAR is difficult to explore, the sizes of the heterocycle and the oxime group are important for the activity. For example, the activity imparted to the 7-(3-aminomethyl-3methylpyrrolidin-1-yl)naphthyridinone ring by the alkyl group of the oxime moiety is as follows: benzyl $>$ ethyl $>$ methyl $(\mathbf{1 0 j} v s \mathbf{1 0 i}$ vs 10h), which suggests that simply increasing the lipophilicity could improve the activity (Table 2). Above all, thiazol-2-yl and 3aminomethyl-4-benzyl oxyimino-3-methylpyrrolidin-1-yl groups are the most active at the N-1 and C-7 positions of naphthyridinone core, respectively.

\section{Table 1}

Structures and in vitro activity of compounds 1a-i and 10a-n against HL60 (at $30 \mu \mathrm{mol} / \mathrm{L}$ )

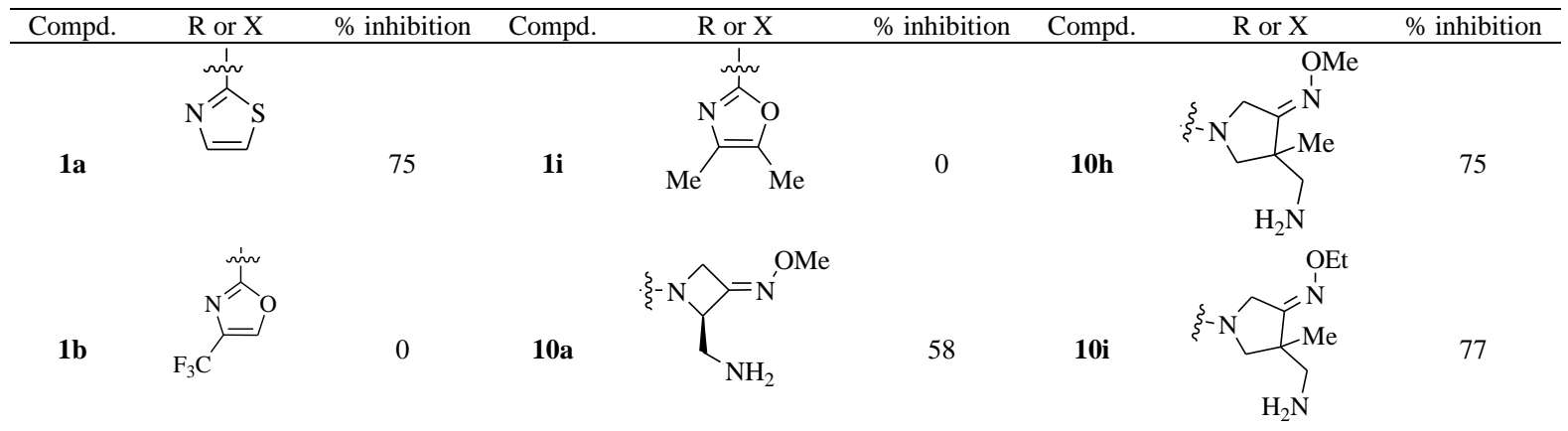


$1 c$<smiles>In1cccc1</smiles>

1d<smiles>CC(C)(C)C1C=CSC=C1</smiles>

10<smiles></smiles>

0<smiles>CC(C)(C)c1ncc[nH]1</smiles>

28<smiles>Nc1ncco1</smiles>

0

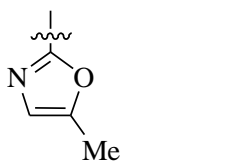

1h

0

$10 \mathrm{~g}$ 10c

$10 b$<smiles>CCO/N=C1\CN(C)C1CN</smiles><smiles>CO/N=C1\CN[C@H](CN)C1</smiles>

10d

$10 \mathrm{e}$

$10 f$<smiles>CCN=C1C=C(OCC)C(CN)C1</smiles>

73

56

9

78

44

10n

$10 \mathrm{j}$

$10 \mathrm{k}$
80<smiles>CO/N=C1\CN(I)CC1(C)N</smiles>

Table 2

In vitro activity of selected compounds against ten cell lines

\begin{tabular}{ccccccccccc}
\hline \multirow{2}{*}{ compd } & HL60 & HepG2 & HCT-116 & A549 & PANC-1 & Hela & DU145 & SKOV3 & MCF-7 & MCF-7/DOX \\
\hline $\mathbf{1 0 c}$ & 1.62 & 16.73 & 39.40 & 17.27 & $>50$ & $>50$ & $>50$ & 49.24 & 35.82 & 16.54 \\
$\mathbf{1 0 e}$ & 0.77 & 13.24 & 27.69 & 11.62 & $>50$ & 32.52 & $>50$ & 28.80 & 24.78 & 23.56 \\
$\mathbf{1 0 h}$ & 12.10 & 15.72 & 23.32 & 22.93 & $>50$ & 42.08 & 26.50 & 22.68 & 31.05 & $<0.5$ \\
$\mathbf{1 0 i}$ & 6.20 & 10.85 & 21.15 & 8.74 & $>50$ & 21.72 & 15.41 & 26.49 & 17.28 & $<0.5$ \\
$\mathbf{1 0 j}$ & 1.18 & 2.99 & 5.62 & 4.05 & 5.30 & 6.25 & 5.52 & 5.18 & 5.57 & $<0.5$ \\
$\mathbf{1 0 k}$ & 2.76 & 13.59 & 29.70 & 16.00 & $>50$ & 44.62 & $>50$ & 25.65 & 19.63 & 1.43 \\
$\mathbf{1 0 m}$ & 4.80 & 6.77 & 12.68 & 5.19 & $>50$ & 15.76 & $>50$ & 34.29 & 12.21 & 26.42 \\
$\mathbf{1 a}$ & 1.17 & 3.17 & 18.74 & 22.06 & $>50$ & 41.64 & 6.96 & 33.90 & 14.59 & 29.21 \\
etoposide & 2.91 & 1.97 & $>50$ & 48.05 & $>50$ & $>50$ & 18.05 & 44.97 & 16.27 & $>50$
\end{tabular}

${ }^{\mathrm{a}} \mathrm{IC}_{50}$ values were presented as the concentration of drug inhibition $50 \%$ cell growth and determined by at least three separate tests and reported

\section{Conclusion}

In summary, structural modifications of compound 1a (a precursor of Voreloxin) at the N-1 position (various heteroaromatic rings instead of the thiazol-2-yl) and C-7 position (four-/five-/six-membered nitrogen heterocyclic amine moieties with an oxime group instead of 3-aminopyrrolidin-1-yl one), respectively, were made in this study. Our results reveal that thiazol-2-yl and 3-aminomethyl-4benzyloxyimino-3- methylpyrrolidin-1-yl groups are optimal at the N-1 and C-7 positions of naphthyridinone core, respectively. The most active compound $\mathbf{1 0 j}$ shows considerable broad-spectrum antitumor activity $\left(\mathrm{IC}_{50}:<0.5-6.25 \mu \mathrm{mol} / \mathrm{L}\right)$ against all of the tested cell lines including Etoposide- and/or 1a-resistant ones and is 1.3->100 fold more potent than those of the two references against these cell lines, except HL60 and HepG2.

\section{Acknowledgment}


This work was supported by National Key Research and Development Program (2016YFA0201500), the National S\&T Major Special Project on Major New Drug Innovations (Nos. 2014ZX09507009-003, 2015ZX09102007) and NSFC (Nos. 81373267, 21502237).

\section{References}

[1] K. Lv, L.L. Wang, X.B. Zhou, et al., Synthesis and in vitro antitumor activity of 1-(3-dimethylamino)propyl indolin-2-one derivatives, Med. Chem. Res. 22 (2013) 1723-1729.

[2] A. Jemal, F. Bray, M.M. Center, et al., Global cancer statistics, Cancer J. Clin. 61 (2011) 69-90.

[3] B.W. Stewart, C.P. Wild, World Cancer Report 2014, World Health Organization, International Agency for Research on Cancer, 2015.

[4] M.S. Kinch, An analysis of FDA-approved drugs for oncology, Drug Discovery Today 19 (2014) 1831-1835.

[5] T.T. Zhang, W.Y. Shen, M.L. Liu, et al., Synthesis, antimycobacterial and antibacterial activity of fluoroquinolone derivatives containing an 3alkoxyimino-4-(cyclopropylanimo)methylpyrrolidine moiety, Eur. J. Med. Chem. 104 (2015) 73-85.

[6] A. Zumla, P. Nahid, S.T. Cole, Advances in the development of new tuberculosis drugs and treatment regimens, Nat. Rev. Drug Discovery 12 (2013) 388404.

[7] A. Ahmed, M. Daneshtalab, Nonclassical biological activities of quinolone derivatives, J. Pharm. Pharm. Sci. 15 (2012) 52-72.

[8] L.S. Feng, M.L. Liu, Advances of structural modifications and nonclassical biological activities of quinolones derivatives, J. Int. Pharm. Res. 37 (2010) $139-143$.

[9] U. Hoch, J. Lynch, Y. Sato, et al., Voreloxin, formerly SNS-595, has potent activity against a broad panel of cancer cell lines and in vivo tumor models, Cancer Chemother. Pharmacol. 64 (2009) 53-65.

[10]J.A. Abbas, R.K. Stuart, Vosaroxin: a novel antineoplastic quinolone, Expert. Opin. Investig. Drugs 21 (2012) $1223-1233$.

[11] Y. Tsuzuki, K. Tomita, K.I. Shibamori, et al., Synthesis and structure-activity relationships of novel 7-substituted 1, 4-dihydro-4-oxo-1-(2-thiazolyl)-1, 8naphthyridine-3-carboxylic acids as antitumor agents. Part 2, J. Med. Chem. 47 (2004) 2097-2109.

[12] K. Lv, Y.X. Sun, L.Y. Sun, et al., Design, synthesis, and in vitro antibacterial activity of fluoroquinolone derivatives containing a chiral 3-(alkoxyimino)-2(aminomethyl)azetidine moiety, ChemMedChem 7 (2012) 1230-1236.

[13]K. Lv, J.W. Wang, M.L. Liu, et al., Synthesis and in vitro antibacterial activity of quinolone/naphthyridone derivatives containing 3-alkoxyimino-4(methyl)aminopiperidine scaffolds, Bioorg. Med. Chem. Lett. 23 (2013) 1754-1759.

[14]H.M. Liu, J. Huang, J.Y. Wang, et al., Synthesis, antimycobacterial and antibacterial evaluation of 1-[(1R, 2S)-2-fluorocyclopropyl]fluoroquinolone derivatives containing an oxime functional moiety, Eur. J. Med. Chem. 86 (2014) 628-638.

[15]J. Huang, H.T. Liu, M.L. Liu, et al., Synthesis, antimycobacterial and antibacterial activity of 1-[(1R, 2S)-2-fluorocyclopropyl]naphthyridone derivatives containing an oxime-functionalized pyrrolidine moiety, Bioorg. Med. Chem. Lett. 25 (2015) 5058-5063.

[16] K. Tomita, Y. Tsuzuki, K.I. Shibamori, et al., Synthesis and structure-activity relationships of novel 7-substituted 1, 4-dihydro-4-oxo-1-(2-thiazolyl))-1, 8naphthyridine-3-carboxylic acids as antitumor agents. Part 1, J. Med. Chem. 45 (2002) 5564-5575.

[17]Z.L. Wan, Y. Chai, M.L. Liu, H.Y. Guo, Improved synthesis of a gemifloxacin intermediate 4-(aminomethyl) pyrrolidin-3-one- $O$-methyloxime dihydrochloride, Chin. J. Med. Chem. 19 (2009) 109-111.

[18]L.S. Feng, M.L. Liu, Y.B. Zhang, H.Y. Guo, New way to synthesize DW286-a novel fluoronaphthyridone antibacterial agent, Chem. Res. Chin. Univ. 27 (2011) 981-983.

[19]X. Guo, Synthesis and antibacterial activity of novel fluoroquinolone derivatives containing substituted pyrrolidyl/piperidyl heterocyclic moieties at 7position, Peking Union Medical College, Beijing, 2011.

[20]C.Y. Hong, Y.K. Kim, J.H. Chang, et al., Novel fluoroquinolone antibacterial agents containing oxime-substituted (Aminomethyl)pyrrolidines: synthesis and antibacterial activity of 7-(4-(aminomethyl)-3-(methoxyimino)pyrrolidin-1-yl)-1-cyclopropyl-6-fluoro- 4-oxo-1, 4-dihydro[1, 8]naphthyridine-3carboxylic Acid (LB20304), J. Med. Chem. 40 (1997) 3584-3593.

[21] Y. Chai, M.L. Liu, B. Wang, et al., Synthesis and in vitro antibacterial activity of novel fluoroquinolone derivatives containing substituted piperidines, Bioorg. Med. Chem. Lett. 20 (2010) 5195-5198.

[22]P. Skehan, R. Storeng, D. Scudiero, et al., New colorimetric cytotoxicity assay for anticancer-drug screening, J. Natl. Cancer Inst. 82 (1990) 1107-1112. 


\section{Graphical Abstract}

Synthesis and in vitro antitumor activity of novel naphthyridinone derivatives

Xue-Dong Jia ${ }^{\text {a, b }}$, Shuo Wang ${ }^{\text {a }}$, Ming-Hua Wang ${ }^{\text {a }}$, Ming-Liang Liu ${ }^{\text {a, }}$, Gui-Min Xia ${ }^{\text {a }}$, Xiu-Jun Liu ${ }^{\text {a }}$, Yun Chai ${ }^{\text {a }}$, Hong-Wei $\mathrm{He}^{\mathrm{a}, *}$

${ }^{a}$ Institute of Medicinal Biotechnology, Chinese Academy of Medical Sciences and Peking Union Medical College, Beijing 100050, China

${ }^{b}$ The First Affiliated Hospital of Zhengzhou University, Zhengzhou 450052, China

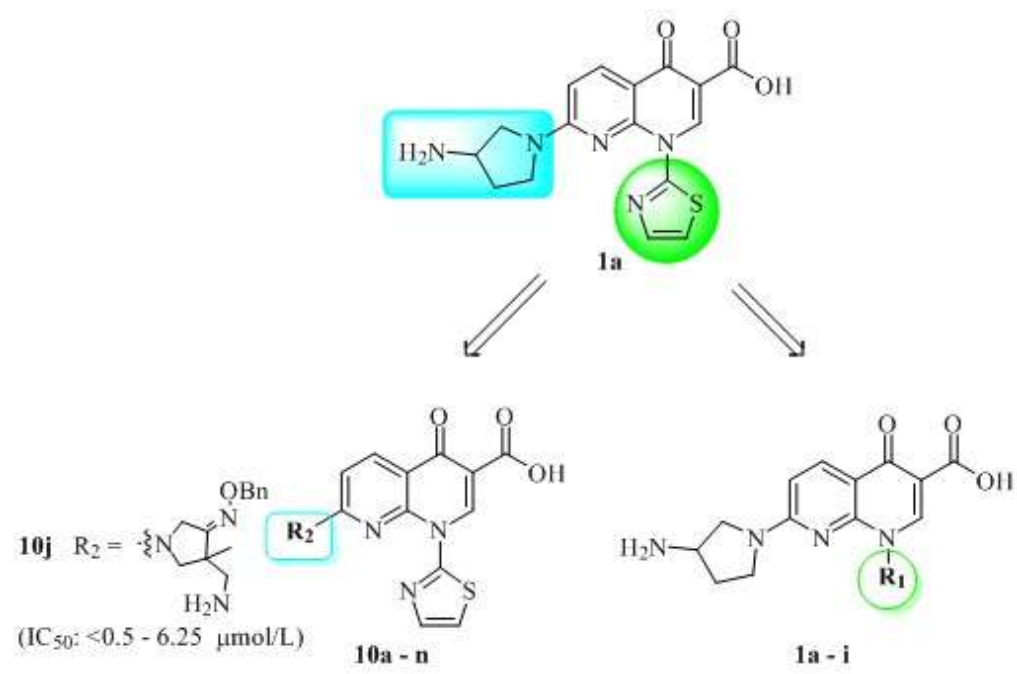

A series of naphthyridinone derivatives based on 1a (a precursor of Voreloxin) were synthesized. 10j exhibits broad-spectrum antitumor activity against all of the cell lines including Etoposide- and/or 1a-resistant ones. 